\title{
DASSO: a data assimilation system for the Southern Ocean that utilizes both sea-ice concentration and thickness observations
}

\section{Letter}

Cite this article: Luo $\mathrm{H}$, Yang $\mathrm{Q}, \mathrm{Mu} \mathrm{L}$, TianKunze X, Nerger L, Mazloff M, Kaleschke L, Chen D (2021). DASSO: a data assimilation system for the Southern Ocean that utilizes both sea-ice concentration and thickness observations. Journal of Glaciology 67(266), 1235-1240. https://doi.org/10.1017/jog.2021.57

Received: 10 October 2020

Revised: 2 May 2021

Accepted: 4 May 2021

First published online: 28 May 2021

\section{Key words:}

Southern Ocean; data assimilation; sea-ice thickness; SMOS

\section{Author for correspondence:}

Qinghua Yang,

E-mail: yangqh25@mail.sysu.edu.cn
Hao Luo ${ }^{1}$, Qinghua Yang ${ }^{1}$ (D), Longjiang $\mathrm{Mu}^{2}$, Xiangshan Tian-Kunze ${ }^{2}$, Lars Nerger ${ }^{2}$, Matthew Mazloff ${ }^{3}$, Lars Kaleschke ${ }^{2}$ and Dake Chen ${ }^{1}$

${ }^{1}$ School of Atmospheric Sciences, Sun Yat-sen University, and Southern Marine Science and Engineering Guangdong Laboratory (Zhuhai), Zhuhai 519082, China; ${ }^{2}$ Alfred Wegener Institute, Helmholtz Centre for Polar and Marine Research, Bremerhaven 27570, Germany and ${ }^{3}$ Scripps Institution of Oceanography, University of California, San Diego, CA, USA

\begin{abstract}
To improve Antarctic sea-ice simulations and estimations, an ensemble-based Data Assimilation System for the Southern Ocean (DASSO) was developed based on a regional sea ice-ocean coupled model, which assimilates sea-ice thickness (SIT) together with sea-ice concentration (SIC) derived from satellites. To validate the performance of DASSO, experiments were conducted from 15 April to 14 October 2016. Generally, assimilating SIC and SIT can suppress the overestimation of sea ice in the model-free run. Besides considering uncertainties in the operational atmospheric forcing data, a covariance inflation procedure in data assimilation further improves the simulation of Antarctic sea ice, especially SIT. The results demonstrate the effectiveness of assimilating sea-ice observations in reconstructing the state of Antarctic sea ice, but also highlight the necessity of more reasonable error estimation for the background as well as the observation.
\end{abstract}

\section{Introduction}

In contrast to the consistent decrease in Arctic sea-ice extent (SIE) during the satellite era, Antarctic SIE exhibited a gradual increase until 2015, but in recent years has abruptly declined (e.g. Stuecker and others, 2017; Turner and Comiso, 2017; Turner and others, 2017; Kusahara and others, 2018; Schlosser and others, 2018; Wang and others, 2019). Although previous studies suggest that trends in Antarctic SIE may result from changes in the atmospheric circulation or ocean stratification (e.g. Hobbs and others, 2016; Jones and others, 2016; Meehl and others, 2019), it is difficult to characterize how Antarctic sea ice is responding to changing climate without reliable sea-ice thickness (SIT) and sea-ice volume (SIV) estimates. Sea-ice data assimilation, merging the information from observations with that from models, can provide more accurate and useful estimates of sea-ice conditions than could otherwise be obtained through either observations or models individually (Buehner and others, 2017).

Due to the success in observing Antarctic sea-ice concentrations (SIC) through satellitebased passive microwave instruments while difficulties in retrieving Antarctic SIT from satellites (Turner and Comiso, 2017), many efforts have been devoted to constraining the simulation of Antarctic sea ice by SIC observations. A simple nudging of model SIC to observation was shown to minimize the effect of observational errors in the interior of the ice pack (e.g. Lindsay and Zhang, 2006). Recently, the SIC observation has also been assimilated by adopting more advanced data assimilation methods such as ensemble Kalman filter (e.g. Massonnet and others, 2013) and 4-D variational analysis (e.g. Mazloff and others, 2010) in Antarctic sea ice-ocean models, which is likely to provide more balanced estimations of model state. However, there are still large uncertainties in the estimation of Antarctic SIT via assimilating SIC only (e.g. Shi and others, 2021). With recent advances in retrieval methods, the soil moisture and ocean salinity (SMOS) satellite data have been used to derive Antarctic SIT, which appears to be more accurate in the thin ice regime (Tian-Kunze and others, 2014; Tian-Kunze and Kaleschke, 2018). As a new kind of observation, although it provides an unique opportunity to investigate the constraint of SIT observations on the Antarctic sea-ice estimates, how to assimilate Antarctic SIT observation derived from SMOS is still debatable.

In this study, a new Data Assimilation System for the Southern Ocean (DASSO) is established based on a regional Southern Ocean sea ice-ocean coupled model, which allows for multivariate model updates in dynamical balance and is convenient to assimilate all available sea-ice observations directly. To the authors' knowledge, this is the first study of assimilating Antarctic SIT observations into a coupled sea ice-ocean model. Thus, based on this developed sea-ice data assimilation system, a unique opportunity exists to evaluate the impact of assimilating SIT together with SIC on Antarctic sea-ice modeling. In addition, because our previous studies suggest that an ensemble of atmospheric forcing can account for atmospheric uncertainties and thus mitigate the need for ensemble inflation in the Arctic sea-ice data assimilation (e.g. Yang and others, 2016a, 2016b; Mu and others, 2018), we would like to see whether this also holds in the Antarctic. 


\section{Methodology}

The DASSO system is based on the Massachusetts Institute of Technology general circulation model (MITgcm, Marshall and others, 1997) and the parallel data assimilation framework (PDAF, Nerger and Hiller, 2013). The model used in this study is a coupled sea ice-ocean model with the same physical configuration as Verdy and Mazloff (2017), which extends from the equator to $78^{\circ} \mathrm{S}$. It has an average $1 / 3^{\circ}$ horizontal grid spacing with 52 unevenly spaced vertical levels from 2.1 to $5800 \mathrm{~m}$. The sea-ice component of model is the viscous-plastic dynamicthermodynamic sea-ice model (Losch and others, 2010). The dynamic part of the sea-ice model is solved by line successive over-relaxation (Zhang and Hibler, 1997) on a C grid, and the thermodynamic counterpart is a 'zero-layer' model (Semtner, 1976). The fifth generation of ECMWF atmospheric reanalyses (ERA5, Hersbach and others, 2020) provides the ensemble of atmospheric forcing as the surface boundary conditions, including air temperature and dewpoint temperature at $2 \mathrm{~m}$, zonal and meridional wind speed at $10 \mathrm{~m}$, surface downward shortwave and longwave radiation flux, surface pressure and total precipitation.

The assimilation scheme adopted in this study is the local error subspace transform Kalman filter (LESTKF, Nerger and others, 2012) provided in PDAF. The LESTKF provides not only consistent projections between the ensemble space and the error subspace, but also minimum transformations of the ensemble members. The solution is given by:

$$
\begin{aligned}
X^{\mathrm{a}}= & \overline{X^{\mathrm{a}}} 1_{m}+X^{\mathrm{a}^{\prime}}=\left[\overline{X^{\mathrm{f}}}+L A(H L)^{\mathrm{T}} R^{-1}\left(y^{\mathrm{o}}-H \overline{X^{\mathrm{f}}}\right)\right] 1_{m} \\
& +X^{\mathrm{f}} \sqrt{m-1} \Omega C \Omega^{\mathrm{T}}
\end{aligned}
$$

where $X$ represents the ensemble model states, $y^{\circ}$ the observation vector, $R$ the observation error covariance matrix and $H$ the observation operator that interpolates from the model grid to observation locations. $L=X^{\mathrm{f}} \Omega$ is the ensemble projected on the error subspace, and $\Omega$ is a projection matrix generated by Householder reflections. $A$ is a transform matrix defined by

$$
A^{-1}=\rho(m-1) I+(H L)^{\mathrm{T}} R^{-1} H L
$$

where $\rho \in(0,1]$ is the forgetting factor used as a tuning parameter of the analysis step to stabilize the filter performance. As $\rho$ decreases, the background error covariance is inflated and results in the reduction of $A$ according to Eqn (2), and finally $X^{\mathrm{a}}$ heads to the observation due to Eqn (1). $C$ is the square root of $A .1_{m}=$ $[1,1, \ldots, 1] \in R^{1 \times m}$. The ensemble size $m$ is 10 here due to only 10 ensemble members available in ERA5. The overbar denotes the ensemble mean. The superscripts a, f, T and ' denote analysis, background, matrix transpose and the ensemble perturbation, respectively. Only horizontal localization is adopted in this study. The localization radius is about $100 \mathrm{~km}$ and the localization function is the Gaspari-Cohn function. To avoid inconsistency introduced by assimilation, we adopt the postprocessing as Tietsche and others (2013) and Kimmritz and others (2018) suggested to ensure the physical consistency of the state of sea-ice and ocean components. Initial perturbations to the ensembles are generated from second-order exact sampling (Pham, 2001) of daily output from a free run of 3 months right before the start of analysis.

The SIT of thin ice from SMOS and the SIC from the Ocean and Sea Ice Satellite Application Facility (OSISAF, Lavergne and others, 2019) are used in data assimilation and, due to a lack of additional data, are also largely used to validate simulations. Operational thin SIT data retrieved from SMOS L-band brightness temperatures (Tian-Kunze and others, 2014) have been disseminated for the Arctic for the time period of 2010 to present. The same retrieval algorithm has been implemented to the Antarctic sea ice, and the product is still under validation (Tian-Kunze and Kaleschke, 2018). Several validation activities have been carried out in the Arctic with promising results for the SMOS SIT product (Tian-Kunze and others, 2014). The uncertainties of the ice thickness increase with increasing ice thickness. The SMOS L-band loses its sensitivity in the thick ice range with thickness more than $1 \mathrm{~m}$. Furthermore, it is found that the retrieved ice thickness is quite sensitive to weather changes because of the immediate thermodynamic equivalence assumption made in the retrieval algorithm (Tietsche and others, 2018). The thickness product is strictly limited to cold periods, i.e. for the Antarctic from April to October. The second version of the SIC interim climate data record (OSI-430-b) has been released by the European Meteorological Satellite Agency for Antarctic as well as Arctic from 2016 onward, which is retrieved from passive microwave instruments on board the Defense Meteorological Satellite Program (DMSP) satellites. Similar to the first version (OSI-409/OSI-409-a), OSI-430-b features an explicit correction of the satellite signal due to weather contamination, dynamic adaptation of algorithm tie points and spatiotemporally varying maps of uncertainties. In addition, OSI-430-b greatly reduces the occurrence of missing data in the final SIC fields due to using all DMSP platforms available at any time, and taking advantage of the overlap of satellite missions. As in our study for the Arctic (e.g. Yang and others, 2016b), the observation error of SIT used in the data assimilation is provided by SMOS, which varies from 0.004 to $0.790 \mathrm{~m}$, whereas that of SIC is a constant value of 0.25 .

To illustrate the impact of sea-ice data assimilation on Antarctic sea-ice simulations and the extent of the model uncertainty as represented by the ensemble spread, three experiments are initialized with the result of Iteration 121 solution of the Biogeochemical Southern Ocean State Estimate (Verdy and Mazloff, 2017) and are conducted over the period from 15 April 2016 to 14 October 2016, which proceeded an unprecedented retreat of Antarctic sea ice in the following austral spring. The control experiment for this study is a free run without data assimilation (denoted Ctrl). Then two experiments that assimilate both SIC and SIT are carried out, with the forgetting factor of one experiment being 1 (denoted F100) and the other being 0.5 (denoted F50). By comparing F100 with F50, we can identify the method to assimilate Antarctic sea-ice observation effectively, which will lay the groundwork for further studies on the Antarctic sea-ice data assimilation.

\section{Results}

The experimental results are examined from both a deterministic and a probabilistic perspective. Figure 1 displays the root mean square error (RMSE) of SIC and SIT against observations. In Ctrl, the RMSE of SIC increases quickly until mid-June and levels off thereafter, whereas that of SIT increases throughout the simulation period. Theses discrepancies between simulations and observations can usually be attributed to model errors and initial errors. Considering the long memory of sea ice, it would be essential to decrease initial errors through data assimilation. Assimilating observational data effectively reduces the RMSE of Antarctic sea-ice simulation at all times. In F100, the RMSE of SIC increases before 17th June and then decreases to 0.088 (Fig. 1a), whereas that of SIT increases until early July and then remains approximately constant (Fig. 1d). The spatial distribution of RMSE differences shows that the data assimilation reduces the SIC error at the edge of sea ice (Fig. 1b) and the SIT error in the Weddell Sea sector as well as 

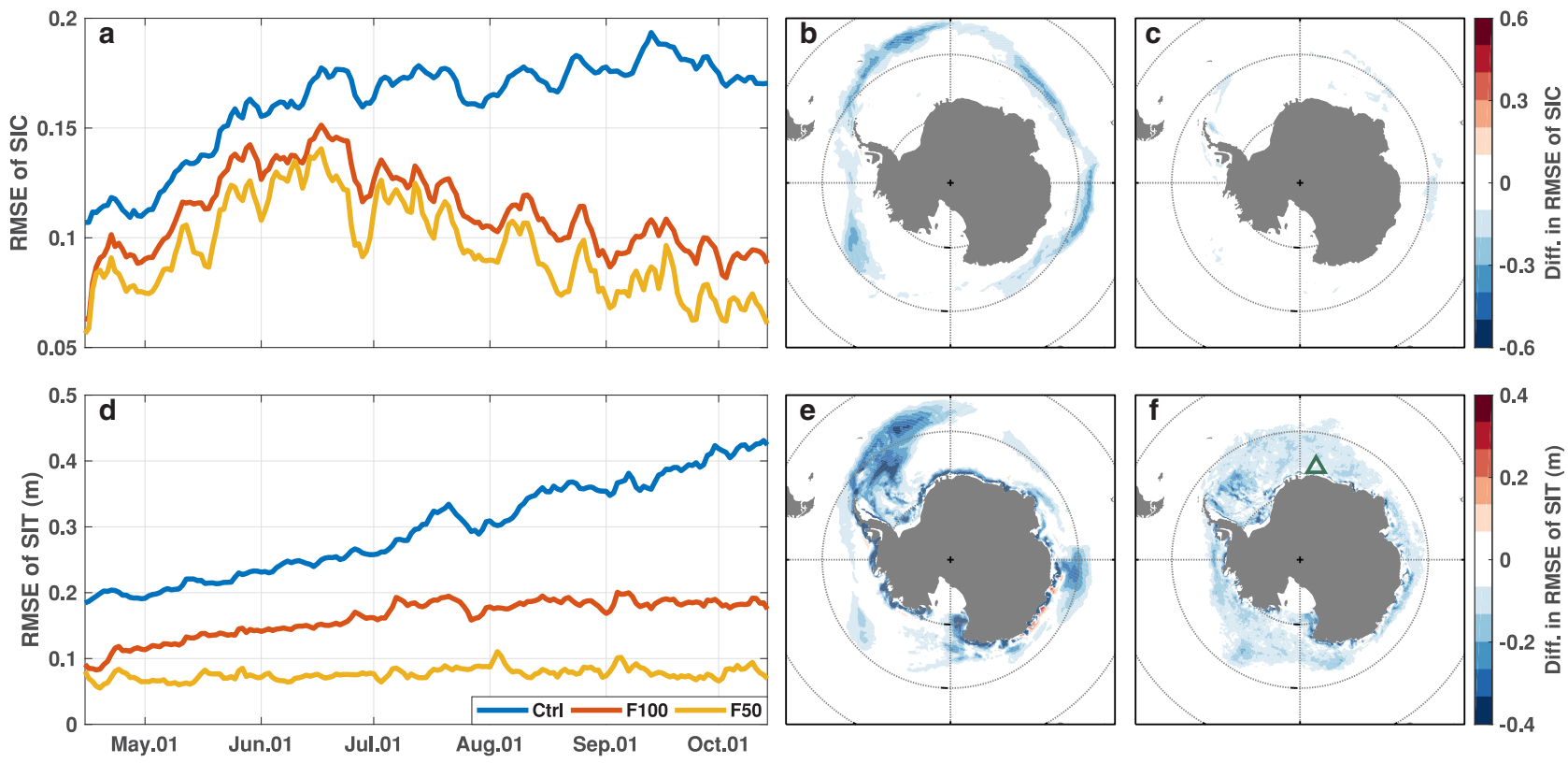

Fig. 1. RMSE of SIC (first row) and SIT (second row, unit: $\mathrm{m}$ ) of the ensemble mean of each experiment compared with the observation. (a, d) The temporal evolution of RMSE. The spatial distribution of RMSE difference (b, e) between F100 and Ctrl, and (c, f) between F50 and F100. The blue, orange and yellow curves in (a, d) denote Ctrl, F100 and F50, respectively. The green triangle in (f) indicates the point $\left(9.8^{\circ} \mathrm{E}, 68.1^{\circ} \mathrm{S}\right)$ chosen for time series analysis in Figs $2 \mathrm{c}$, d.

coastal areas of the Antarctic continent (Fig. 1e). These improvements stress the impact of data assimilation on the Antarctic sea-ice modeling. The RMSE of F50 is less than that of F100 as expected, but there are differences in the RMSE reduction between SIC and SIT. First, the difference in RMSE between F50 and F100 is greater for SIT than for SIC. Second, in F50, the RMSE of SIT stays small with no trend (Fig. 1d), whereas that of SIC behaves similarly to F100 (Fig. 1a). Third, in terms of spatial distribution, F50 improves over F100 in the Southern Ocean for SIT (Fig. 1f), but not much for SIC (Fig. 1c).

To quantify the difference between F100 and F50, the analysis increment vector (increment $=H \overline{X^{\mathrm{a}}}-H \overline{X^{\mathrm{f}}}$ ) is projected onto the innovation vector (innovation $=y^{\mathrm{o}}-H \overline{X^{\mathrm{f}}}$ ), which is defined as

$$
\text { projection }=\frac{\text { increment } \cdot \text { innovation }}{\mid \text { innovation }\left.\right|^{2}}
$$

where the dot between increment and innovation denotes inner product, and $\|$ denotes the norm of the vector. This projection determines to what extent the observation information is absorbed in DASSO. As shown in Figs 2a, b, the projections of SIC and SIT are similar in F100. The projection of SIC (SIT) decreases rapidly in the first 4 (14) analysis cycles, and then remains low until the end of the experiment, suggesting that the majority of observation information absorbed in F100 is from the first few analysis cycles. Combined with what has been shown in Fig. 1, this result indicates that Antarctic sea ice is of long memory, and initial condition should be important for its simulation and prediction. The evolutions of SIC and SIT projection are quite different in F50. The projection of SIC decreases rapidly in the beginning, then decreases slowly before mid-June, followed by a gradual increase toward the end of experiment. Meanwhile, the projection of SIT stays at a relatively high level. These discrepancies result in different RMSE evolutions of SIC and SIT in F50 (Figs 1a, d).

To further illustrate the influence of data assimilation on sea-ice simulation, Figs $2 c$, d show the evolution of SIC and SIT at $\left(9.8^{\circ} \mathrm{E}, 68.1^{\circ} \mathrm{S}\right)$, where both variables have considerable variability and the observed SIT is always $<1 \mathrm{~m}$ during the experiment. Compared with Ctrl and F100, F50 can reproduce the high-frequency fluctuation of SIC and SIT from OSISAF and
SMOS. The observed high-frequency fluctuation of sea ice may be owed to atmospheric processes on the synoptic time scale, whereas this fluctuation is not captured in Ctrl due to limitations of understanding and modeling the air-ice-sea interaction (Swart and others, 2019). Besides, obvious differences in the sea-ice evolution between F100 and F50 can be attributed to whether a covariance inflation procedure is adopted in the assimilation, which can directly affect the probability distribution of ensemble model states.

To evaluate whether a collection of ensemble model states for a scalar variable satisfies the consistency condition, Talagrand diagrams for SIC and SIT are shown in Figs 3a, b. The Talagrand diagram is generated by ranking the values of $m$ ensemble members in increasing order, which divides the range of target variable into $m+1$ intervals, and then the value of the verifying observation falls into one of the $m+1$ categories. Talagrand diagrams of Ctrl and F100 both show a U-shaped distribution, suggesting that the ensemble members tend to be too similar to each other but different from the observation. Thus, the ensembles of Ctrl and F100 are underdispersed. The Talagrand diagram of F50 is rather flat, indicating that the probability distribution of observations is quite well represented by the ensemble. In addition, there is an overpopulation of the smallest ranks in the Talagrand diagram of Ctrl, implying that both SIC and SIT are overestimated in Ctrl. This overestimation is reduced in the assimilation experiments, especially in F50.

The temporal evolution of Antarctic SIE and SIV is shown in Figs $3 c$, d. The SIE is calculated as the sum of the area of all gridcells with SIC $>15 \%$. The SIV is calculated as the sum of the volume of all gridcells with SIT $<1 \mathrm{~m}$ because of the saturation of SMOS brightness temperatures over thicker ice (Tian-Kunze and others, 2014). The observed SIE increases gradually, and reaches a maximum on 27th August. In contrast, the observed SIV increases slowly during the whole simulation period. The evolution of SIE and SIV in Ctrl is similar to the observations, but there are significant positive biases, which are reduced in the assimilation experiments, especially in F50. However, although assimilating sea-ice observations reduces RMSEs of SIV and SIE, there are differences between SIV and SIE in terms of the anomaly correlation coefficient (ACC). Compared to Ctrl, the 

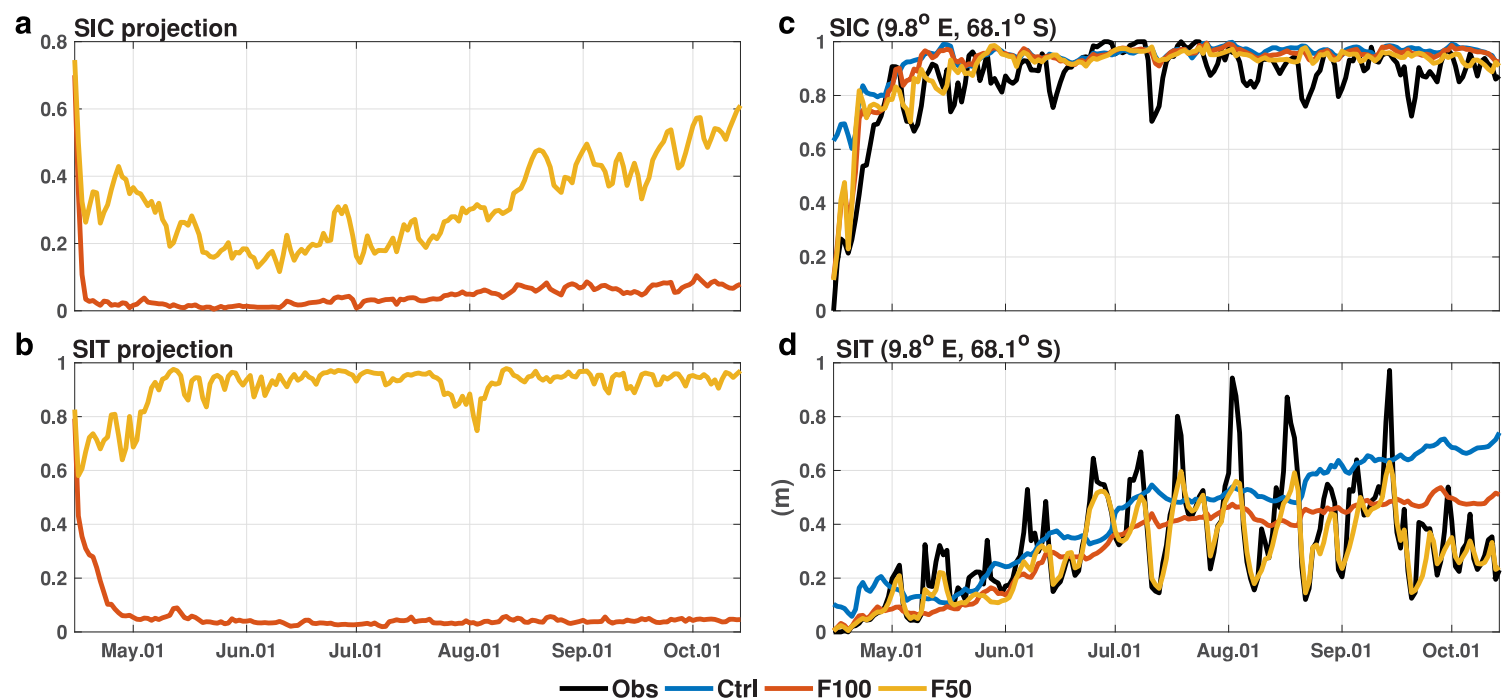

Fig. 2. Coefficient of projecting analysis increment onto innovation for (a) SIC and (b) SIT. And the time series of (c) SIC and (d) SIT (unit: $\mathrm{m}$ ) at $9.8^{\circ} \mathrm{E}, 68.1^{\circ} \mathrm{S}$ in the observation as well as in the ensemble mean of simulation. The black, blue, orange and yellow curves denote observation, Ctrl, F100 and F50, respectively.

a

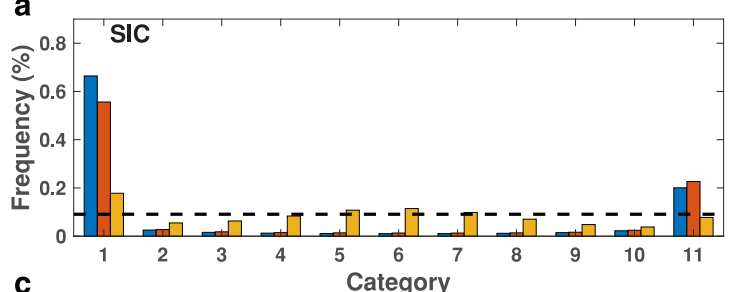

C

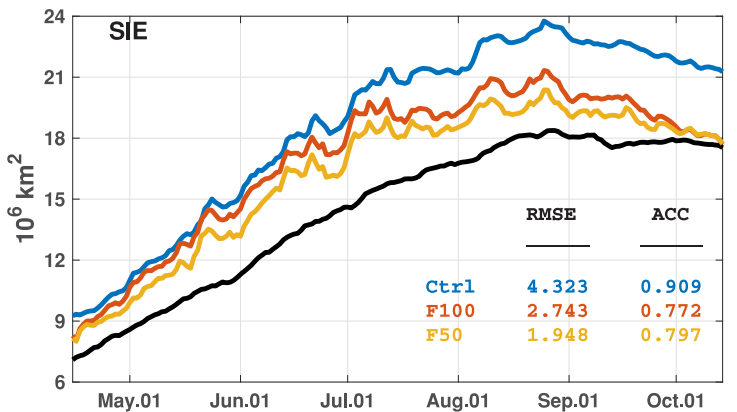

b
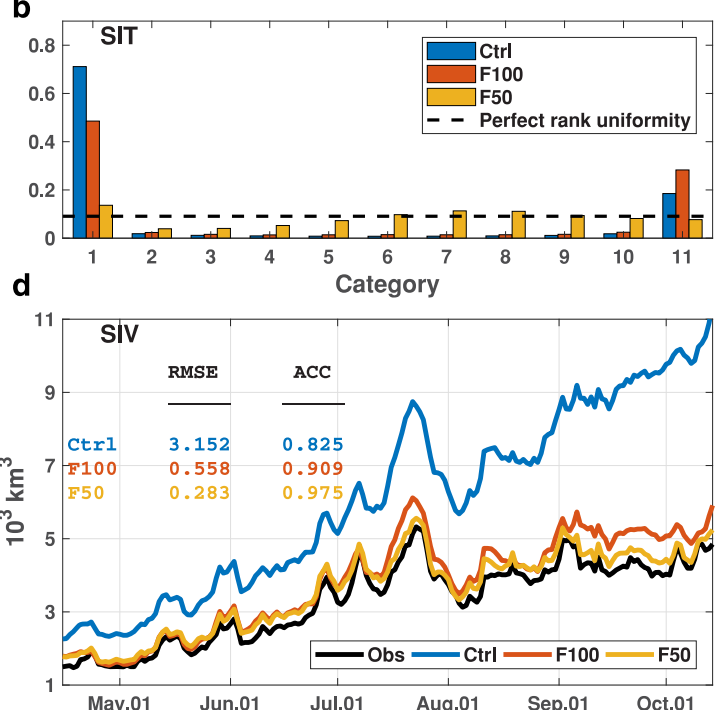

Fig. 3. Talagrand diagrams of (a) SIC and (b) SIT. The dashed line indicates the perfect rank uniformity (i.e. 1/11). The temporal evolution of (c) SIE (unit: $10^{6} \mathrm{~km}^{2}$ ) and (d) SIV (unit: $10^{3} \mathrm{~km}^{3}$ ) in the observations as well as in the ensemble mean of the simulations. The corresponding RMSE and ACC of each experiment compared with observations are also displayed. The black, blue, orange and yellow colors denote observation, Ctrl, F100 and F50, respectively.

ACC of SIV is increased, whereas that of SIE is decreased, in the assimilation experiments. It is worth noting that SIC (SIT) observations play an important role in the simulation of SIE (SIV) during analysis cycles, and the only difference in configuration between assimilating SIC and assimilating SIT is the choice of the observation error in this study. Thus, the differences in ACC between SIE and SIV might result from different choices of observation error for SIC and SIT.

To further validate the performance of DASSO, independent observations are required. And the observation platform for independent observation should be different from that of assimilated observation. However, there is the lack of independent data during assimilation experiments (i.e. from 15 April to 14 October, 2016), whereas in situ sea-ice observations provided by the Antarctic Sea Ice Processes \& Climate program (ASPeCt) are available from 4 to 23 November 2016 (Fig. 4a). Hence, experiments are extended from 15 October to 23 November, during which satellite-based observations have not been assimilated anymore. The correlations between simulation and ASPeCt are low, due to the difference in spatial resolution between simulation $\left(1 / 3^{\circ}\right)$ and ASPeCt $(\sim 11.1 \mathrm{~km})$ and significant variation of sea ice over horizontal distances as small as a few meters. Thus, bias and RMSE will be proper metrics to compare simulation with ASPeCt, which reflects the average feature of the navigation area. As Figs 4d, e show, biases and RMSE in experiments initialized with assimilating sea-ice observation (i.e. F100 and F50) can be suppressed effectively, compared with that without assimilation (i.e. Ctrl). Besides, compared to F100, F50 can further reduce biases and RMSE in sea-ice simulation, especially in the simulation of SIT. These results suggest that Antarctic sea ice is of long memory, and the more reasonable scheme of sea-ice data assimilation will play an essential role in the simulation as well as in the prediction of Antarctic sea ice.

\section{Concluding remarks}

This study introduces a data assimilation system called DASSO based on a regional Southern Ocean sea ice-ocean coupled 

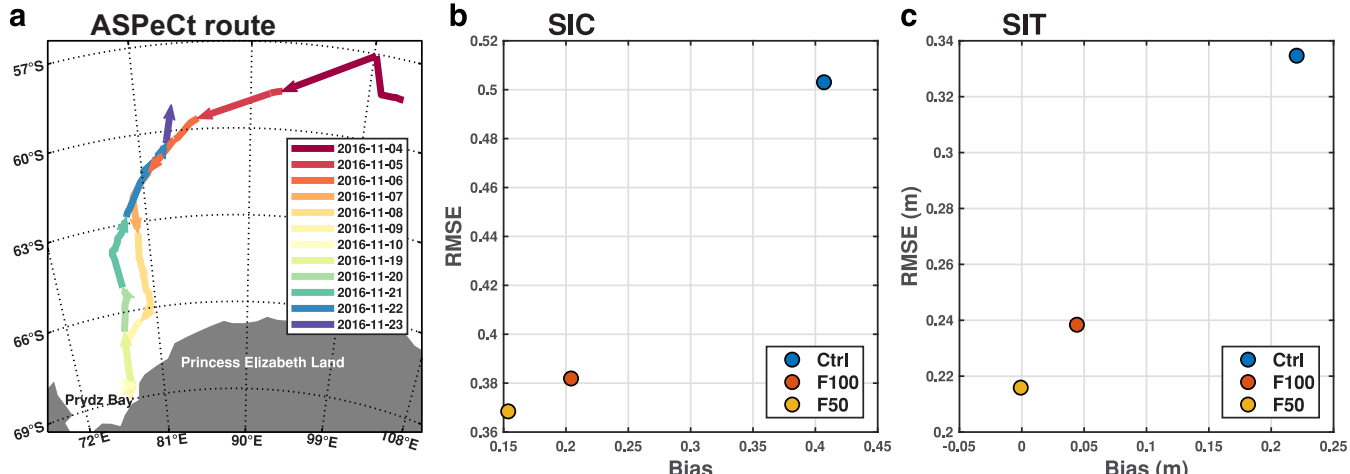

Fig. 4. (a) Route of ASPeCt from 4 to 23 November 2016. The bias and RMSE of (b) SIC and (c) SIT, with respect to ASPeCt. Arrows of different colors in (a) represent navigation directions in different dates. The blue, orange and yellow in (b, c) represent Ctrl, F100 and F50, respectively.

model, and presents a set of assimilation experiments to assess the impact of SIT as well as SIC observations on reproducing the sea-ice conditions and variations during the period from 15 April to 14 October, 2016. Generally, assimilating SIC and SIT improves the simulation of Antarctic sea ice, and in particular suppresses the positive biases of SIT and SIC in the model-free run. Even though atmospheric uncertainties are partially accounted for by using the ERA5 atmospheric state ensemble, ERA5 ensemble is usually underestimated because ERA5 ensemble takes mostly random uncertainties into account, while systematic model errors are not considered (Hersbach and others, 2020). Therefore, the uncertainty in modeled sea ice is also underestimated in the model ensemble, which leads to filter divergence eventually. Thus, how to fully consider the model uncertainty is of great importance in sea-ice data assimilation. Although Yang and others (2016a) and Mu and others (2018) found it is unnecessary to use additional inflation if the UKMO ensemble forcing is applied in the Arctic sea-ice data assimilation, here a covariance inflation procedure is necessary in the Antarctic sea-ice data assimilation to counteract the underestimation of ensemble variance resulted from underdispersed ERA5 ensemble, which significantly improves the simulation of Antarctic sea ice and lays the groundwork for further studies on the Antarctic sea-ice data assimilation. This also suggests that there are obvious differences in sea-ice data assimilation between the two poles, and the experience of Arctic sea-ice data assimilation cannot be simply transplanted to the Antarctic.

The results described here represent our first attempt to develop a new DASSO to improve Antarctic sea-ice simulation and estimation. Given the limitations of the present Antarctic SIT reanalysis data (Shi and others, 2021), further refinements and extensions are very much needed. For example, the difference between SIC and SIT RMSE in the assimilation experiments suggests that the observation error should be estimated in a more reasonable way. In addition, it is important to isolate the impact of different sea-ice observations on reproducing Antarctic sea iceocean variability. In future research, we would like to address this problem systematically based on DASSO. On a final note, since the experimental Antarctic EnviSat-2 and CryoSat-2 SIT data have been recently released, although with large uncertainties (Hendricks and others, 2018), and since the Antarctic ICESat-2 SIT data are also due to be released in the near future (Kacimi and Kwok, 2020), the prospects look bright for reconstructing long-term Antarctic SIT and volume through sea-ice data assimilation.

Acknowledgements. The authors thank two anonymous reviewers for their very helpful comments and suggestions. We thank Petra Heil of Australia Antarctic Division and Australian Antarctic Program Partnership for providing ASPeCt sea-ice observations from 4 to 23 November 2016. We also thank Guokun Lyv of Shanghai Jiao Tong University and Chao-Yuan Yang of Sun Yat-sen University for useful discussions. This is a contribution to the Year of Polar Prediction (YOPP), a flagship activity of the Polar Prediction Project (PPP), initiated by the World Weather Research Programme (WWRP) of the World Meteorological Organisation (WMO). We acknowledge the WMO WWRP for its role in coordinating this international research activity. This study is supported by the National Natural Science Foundation of China (Nos. 41941009, 41922044 and 42006191), and the Guangdong Basic and Applied Basic Research Foundation (No. 2020B1515020025). SMOS Sea Ice Thickness data is produced within the framework of 'SMOS sea ice thickness processing and dissemination service' supported by ESA under the contract No. 4000i2473 1/18/I-EF. We thank the National Supercomputer Center in Guangzhou for providing compute resources.

\section{References}

Buehner M, Bertino L, Caya A, Heimbach P and Smith G (2017) Sea Ice data assimilation. In Lemieux J-F, Toudal Pedersen L, Buehner M and Carrieres $\mathrm{T}$ (eds), Sea Ice Analysis and Forecasting: Towards an Increased Reliance on Automated Prediction Systems. Cambridge, Cambridge University Press, pp. 51-108.

Hendricks S, Paul S and Rinne E (2018) ESA Sea Ice Climate Change Initiative (Sea_Ice_cci): Southern hemisphere sea ice thickness from the CryoSat-2 satellite on a monthly grid (L3C), v2.0. Centre for Environmental Data Analysis.

Hersbach $\mathbf{H}$ and 42 others (2020) The ERA5 global reanalysis. Quarterly Journal of the Royal Meteorological Society 146(730): 1-51. doi: 10.1002/ qj.3803

Hobbs WR and 5 others (2016) A review of recent changes in Southern Ocean sea ice, their drivers and forcings. Global and Planetary Change 143, 228-250. doi: 10.1016/j.gloplacha.2016.06.008

Jones JM and 24 others (2016) Assessing recent trends in high-latitude southern hemisphere surface climate. Nature Climate Change 6(10), 917-926. doi: $10.1038 /$ nclimate 3103

Kacimi S and Kwok R (2020) The Antarctic sea ice cover from ICESat-2 and CryoSat-2: freeboard, snow depth and ice thickness. The Cryosphere 14, 4453-4474. doi: 10.5194/tc-14-4453-2020

Kimmritz M and 5 others (2018) Optimising assimilation of sea ice concentration in an earth system model with a multicategory sea ice model. Tellus A: Dynamic Meteorology and Oceanography 70(1), 1-23. doi: 10.1080/ 16000870.2018.1435945

Kusahara K, Reid P, Williams GD, Massom R and Hasumi H (2018) An ocean-sea ice model study of the unprecedented Antarctic sea ice minimum in 2016. Environmental Research Letters 13(8), 084020. doi: 10.1088/ 1748-9326/aad624

Lavergne T and 16 others (2019) Version 2 of the EUMETSAT OSI SAF and ESA CCI sea-ice concentration climate data records. The Cryosphere 13(1), 49-78. doi: 10.5194/tc-13-49-2019

Lindsay RW and Zhang J (2006) Assimilation of ice concentration in an iceocean model. Journal of Atmospheric and Oceanic Technology 23(5), 742749. doi: $10.1175 /$ jtech 1871.1 
Losch M, Menemenlis D, Campin J-M, Heimbach P and Hill C (2010) On the formulation of sea-ice models. Part 1: effects of different solver implementations and parameterizations. Ocean Modelling 33(1-2), 129-144. doi: 10.1016/j.ocemod.2009.12.008

Marshall J, Adcroft A, Hill C, Perelman L and Heisey C (1997) A finitevolume, incompressible Navier Stokes model for studies of the ocean on parallel computers. Journal of Geophysical Research: Oceans 102(C3), 5753-5766. doi: 10.1029/96jc02775

Massonnet F and 6 others (2013) A model reconstruction of the Antarctic sea ice thickness and volume changes over 1980-2008 using data assimilation. Ocean Modelling 64, 67-75. doi: 10.1016/j.ocemod.2013.01.003

Mazloff MR, Heimbach $\mathbf{P}$ and Wunsch C (2010) An eddy-permitting Southern Ocean state estimate. Journal of Physical Oceanography 40(5), 880-899. doi: 10.1175/2009jpo4236.1

Meehl GA and 7 others (2019) Sustained ocean changes contributed to sudden Antarctic sea ice retreat in late 2016. Nature Communications 10(1), 14. doi: 10.1038/s41467-018-07865-9

Mu L and 6 others (2018) Improving sea ice thickness estimates by assimilating CryoSat-2 and SMOS sea ice thickness data simultaneously. Quarterly Journal of the Royal Meteorological Society 144(711), 529-538. doi: 10.1002/qj.3225

Nerger L and Hiller W (2013) Software for ensemble-based data assimilation systems - implementation strategies and scalability. Computers \& Geosciences 55, 110-118. doi: 10.1016/j.cageo.2012.03.026

Nerger L, Janjić T, Schröter J and Hiller W (2012) A unification of ensemble square root Kalman filters. Monthly Weather Review 140(7), 2335-2345. doi: $10.1175 / \mathrm{mwr}-\mathrm{d}-11-00102.1$

Pham DT (2001) Stochastic methods for sequential data assimilation in strongly nonlinear systems. Monthly Weather Review 129(5), 1194-1207. doi: 10.1175/1520-0493(2001)129<1194:Smfsda>2.0.Co;2

Schlosser E, Haumann FA and Raphael MN (2018) Atmospheric influences on the anomalous 2016 Antarctic sea ice decay. The Cryosphere 12(3), 1103-1119. doi: 10.5194/tc-12-1103-2018

Semtner Jr AJ (1976) A model for the thermodynamic growth of sea ice in numerical investigations of climate. Journal of Physical Oceanography 6 (3): 379-389. doi: 10.1175/1520-0485(1976)006<0379:Amfttg>2.0.Co;2

Shi Q and 5 others (2021) Evaluation of sea-ice thickness from four reanalyses in the Antarctic Weddell Sea. The Cryosphere 15(1), 31-47. doi: 10.5194/ tc-15-31-2021

Stuecker MF, Bitz CM and Armour KC (2017) Conditions leading to the unprecedented low Antarctic sea ice extent during the 2016 austral spring season. Geophysical Research Letters 44(17), 9008-9019. doi: 10.1002/ $2017 \mathrm{gl} 074691$

Swart S and 19 others (2019) Constraining Southern Ocean air-sea-ice fluxes through enhanced observations. Frontiers in Marine Science 6, 421. doi: 10. 3389/fmars.2019.00421

Tian-Kunze X and Kaleschke L (2018) SMOS-derived thin sea ice thickness in the Arctic and Antarctic. ISAR-5, Tokyo, Japan, ISAR-5.

Tian-Kunze X and 6 others (2014) SMOS-derived thin sea ice thickness: algorithm baseline, product specifications and initial verification. The Cryosphere 8(3), 997-1018. doi: 10.5194/tc-8-997-2014

Tietsche S, Notz D, Jungclaus J and Marotzke J (2013) Assimilation of sea-ice concentration in a global climate model - physical and statistical aspects. Ocean Science 9(1), 19-36. doi: 10.5194/os-9-19-2013

Tietsche S and 5 others (2018) Thin Arctic sea ice in L-band observations and an ocean reanalysis. The Cryosphere 12(6), 2051-2072. doi: 10.5194/ tc-12-2051-2018

Turner J and Comiso J (2017) Solve Antarctica's sea-ice puzzle. Nature 547 (7663), 275-277. doi: 10.1038/547275a

Turner J and 6 others (2017) Unprecedented springtime retreat of Antarctic sea ice in 2016. Geophysical Research Letters, 44(13): 6868-6875. doi: 10. 1002/2017gl073656

Verdy A and Mazloff MR (2017) A data assimilating model for estimating Southern Ocean biogeochemistry. Journal of Geophysical Research: Oceans 122(9), 6968-6988. doi: 10.1002/2016jc012650

Wang Z, Turner J, Wu Y and Liu C (2019) Rapid decline of total Antarctic sea ice extent during 2014-16 controlled by wind-driven sea ice drift. Journal of Climate 32(17), 5381-5395. doi: 10.1175/ jcli-d-18-0635.1

Yang Q, Losch M, Losa SN, Jung T and Nerger L (2016a) Taking into account atmospheric uncertainty improves sequential assimilation of SMOS sea ice thickness data in an ice-ocean model. Journal of Atmospheric and Oceanic Technology 33(3), 397-407. doi: 10.1175/ jtech-d-15-0176.1

Yang Q and 5 others (2016b) Brief communication: the challenge and benefit of using sea ice concentration satellite data products with uncertainty estimates in summer sea ice data assimilation. The Cryosphere 10(2), 761-774. doi: $10.5194 /$ tc-10-761-2016

Zhang J and Hibler WD III (1997) On an efficient numerical method for modeling sea ice dynamics. Journal of Geophysical Research 102(C4), 8691-8702. doi: 10.1029/96jc03744 